\title{
Palladium nanofiber networks hydrogen sensor and hydrogen-actuated switches
}

\author{
Boyi wang ${ }^{1}$, Yong Zhu ${ }^{1}$, Vien Huynh ${ }^{2}$,Md.Ataur Rahman ${ }^{3}$, Brian Hawkett ${ }^{2}$, Sharath \\ Sriram $^{3}$,Dzung Viet Dao ${ }^{1}$ \\ ${ }^{1}$ Queensland Mirco-and Nanotechnology Centre, Griffith University, Nathan, QLD 4111, \\ Australia \\ ${ }^{2}$ Key Centre for Polymers and Colloids, School of Chemistry and University of Sydney Nano \\ Institute, The University of Sydney, NSW 2006, Australia \\ ${ }^{3}$ Functional Materials and Microsystems Research Group and the Micro Nano Research \\ Facility, RMIT University, Melbourne, Australia
}

\begin{abstract}
This paper presents a novel fabrication technique for hydrogen gas sensor based on polymer nanofiber and pure palladium metal. The polymer nanofiber was produced from $7 \mathrm{wt} \%$ poly vinyl alcohol (PVA) solution through electrospinning system. The multilayer PVA nanofibers was utilized as a substrate to create a networks of palladium nanofibers hydrogen sensor. The palladium nanofiber with a thickness of $30 \mathrm{~nm}$ was deposited on the surface of the multilayer PVA nanofibers by using E-beam evaporation system and shadow mask. During the hydrogen detection test, the novel hydrogen sensor was presented as a set of hydrogen-actuated switches with high sensitivity. The fabrication process was simple and inexpensive, and the sensor can be massproduced.
\end{abstract}

Keywords: Nanofiber, Poly vinyl Alcohol, Palladium, Hydrogen sensor, switches

\section{Introduction}

Hydrogen $\left(\mathrm{H}_{2}\right)$, as a clean combustible gas, has a very low density and boiling point combined with a high diffusion coefficient and buoyancy. Furthermore, as a combustible gas, it has a low minimum ignition energy, high heat of combustion and wide flammable range, as well as a high ignition temperature [1]. As a dangerous flammable gas in the world, the hydrogen gas is colorless, odorless and tasteless. In terms of its physical characteristics, it cannot be detected by human senses. Based on this critical aspect, fast, economical and reliable detection of hydrogen gas is required for hydrogen-based application and safety improvement [2].

In recent years, hydrogen gas sensors have been improved in response speed, sensitivity, and cost. Miniature hydrogen sensing materials have been a major field in the development for these new generation of hydrogen gas sensor due to their large surface area to volume (SA/A) ratios, which is able to enhance the absorption and 
desorption rates of the sensing materials, and improve the hydrogen diffusion rate in the sensing materials [3]. Conventionally, Metal oxide hydrogen sensing materials are widely used for hydrogen sensors fabrication in the past couple of years. However, these sensing materials can detect various other common combustible gas during the gas detection process, which reduces selectivity and sensitivity for hydrogen gas when they are exposed to air at room temperature and atmospheric pressure. In addition, electrometry has also been used in mass spectrometry for chemical analysis [4-6]. However, the overall spectrometry system is fairly complicated and costly. As a promising hydrogen sensing material, palladium (Pd)-based hydrogen sensors have proved to a significant research filed in the past several years [7].

Palladium is commonly unitized as a hydrogen sensing material for hydrogen gas detection and hydrogen concentration measurement. The pure palladium has a unique ability to absorb up to 900 times its own volume of hydrogen. The absorption of hydrogen molecule by pure palladium can form Pd-H hydride, and hydrogen atoms are able to occupy the interstitial sites of the Pd lattice. Based on this phenomenon, the lattice of Pd is expanded, and leading to changes of its physical properties, which include volume, reflectivity, refractive index, and electrical resistivity. Hydrogen is desorbed when Pd under non-equilibrium condition, thus the concentration of hydrogen in the Pd is higher than that in the surface. Based on the concentration gradient of hydrogen, the chemical bonds between Pd and hydrogen atoms in the Pd metal are broken and the hydrogen atoms move to the surface of Pd, and are desorbed to the air. In order to achieve high activation energy to break Pdf-H bonds, the concentration gradient is sufficiently high enough to provide the necessary driving force [8]. Based on the previous research, the pure palladium film is easy to bubble and crack after absorbing and desorbing hydrogen for several times [9], which can negatively influence the stability and reliability of hydrogen detection of the palladium-based hydrogen sensor. Therefore, Mechanical stress in the film and lattice expansion of palladium structure are the significant issues for further research to address [10].

In literature, there are two solutions that are able to address the crack of pure palladium film after absorbing and desorbing hydrogen. The first method is to expand the lattice structure of palladium before absorbing and desorbing hydrogen gas. The second method is to fabricate low-dimensional structures, such as nanofiber and nanoparticle to avoid the fracture issue of pure palladium film. Thanks to the quantum effects, $0 \mathrm{D}$, 1D, and 2D structures have unique properties which can be utilized to improve senso's performance [11,12]. The first method investigated previously [10] indicated that the lattice structure of palladium was significant expanded after doping yttrium atoms with palladium. Due to the improvement of the lattice structure, the palladium-yttrium alloy thin film was able to enhance the response rate and eliminate the bubble and delamination phenomena. However, the pathway of hydrogen absorption and desorption was limited due to hydrogen atoms were only available through surface to bottom. Meanwhile, the response and recovery speed of the hydrogen sensor has been directly influenced. 
The low-dimensional structure of palladium-based hydrogen sensor is the focus of this paper. Conversion of materials into nanoscale reduces crystal defects, thereby enhancing mechanical strength and reducing the fracture phenomenon. Conventional method introduced by Offermans et al [13] utilized a technique called deposition and etching under angles (DEA) to produce the single palladium nanowire hydrogen sensor with fast response speed, but the DEA technique was difficult to be controlled, and expensive for mass-production. Lim et al [14] have reported a highly sensitive hydrogen sensor based on a single palladium-functionalized carbon nanowire suspended about 10 um above a substrate while monolithically bridging two carbon posts. The palladium nanowire was fabricated based on carbon-microelectromechanical system process. While this hydrogen sensor has been reported to achieved high sensitively and fast response, the complex fabrication process was proved not suitable for the application. Zeng et al [15] did research on hydrogen gas sensing with networks of palladium nanowires formed on filtration membranes. Using the surface structure of filtration membranes as a substrate to realize the palladium nanowires. The fabrication process was simple and inexpensive, but the hydrogen response speed and sensitivity was negatively influenced due to the diameter of palladium nanowires been limited by the filtration membranes. Collectively, the palladium nanofiber is of great complexity to fabricate and it normally require expensive nano-fabrication facilities for massproduction.

In this paper, we present a novel fabrication technique for hydrogen gas sensor, which was based on polymer nanofiber and pure palladium metal. The polymer nanofiber was produced by electrospinning system and poly vinyl alcohol (PVA) solution. The multilayer PVA nanofibers were fabricated as a substrate for the hydrogen sensor. The networks of palladium nanofibers formed on the unique PVA substrate. The fabrication process of the suspended Pd/PVA nanofibers based hydrogen sensor was simple and has fewer steps by utilized shadow mask and E-beam evaporation system. The proposed fabrication process was suitable for mass-production of the sensor. The microstructure of the nanofibers were determined via high-resolution microscopy, and scanning electron microscope (SEM). The proposed hydrogen sensor was tested to have an interesting resistance trend when it was exposed to hydrogen gas.

\section{$2 \quad$ Experiments}

\subsection{Multilayer PVA nanofiber substrate preparation}

Electrospinning is a distinctive technique that allows the fabrication of continuous fibres with diameters ranging from submicro to nanoscale in either single or multilayer. Therefore, the polymer nanofibers can be produced to achieve a thinner diameter and a larger surface area than conventional techniques. As the schematic diagram shown in Fig. 1, the electrospinning system (Spraybase ${ }^{\circledR}$ Electrospinning kit) consists of three major components: a high voltage supply, a syringe with a needle, and a grounded collector plate. The needle was connected to the high voltage supply that is capable to generate DC voltages up to $30 \mathrm{KV}$. The morphology and diameter of fibre is controlled 
by intrinsic properties of the polymer solution and the operation conditions during the electrospinning process. Thus, the types of polymer, concentration of the solvent, and electrical conductivity are critical in determining the morphology and diameter of fibres. The electrospinning system has to operate under high voltage, which is dangerous for operators and bystanders. Thus, the high voltage sealed chamber must be closed during the operation process.

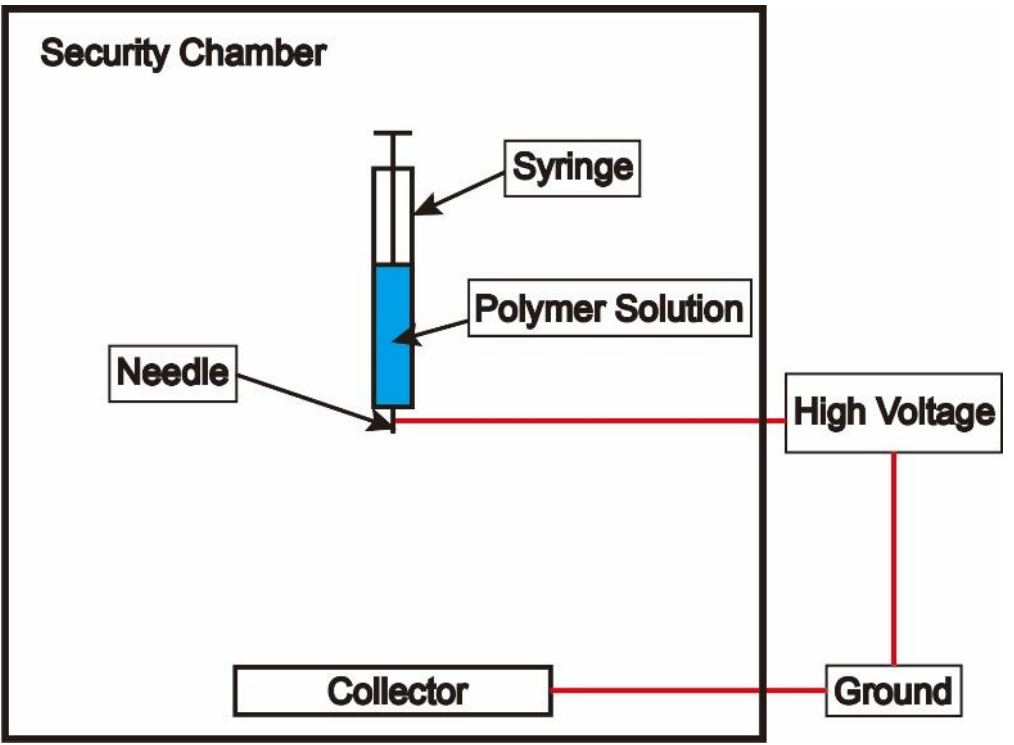

Fig. 1. Schematic illustration of the setup for electrospinning system

The 7 wt $\%$ PVA solution was produced before the PVA nanofibers fabrication. The PVA solution was continuously supplied using a syringe pump at a rate of $10 \mathrm{uL} / \mathrm{min}$. A voltage of $7 \mathrm{KV}$ was applied on the needle. The distance between needle and collector plate was $7 \mathrm{~cm}$. the particular glass substrate was prepared before the electrospinning process, as shown in Fig. 2(a). Based on the material characteristics of PVA nanofibers, the soft and light nanofibers are difficult to be transferred from the collector plate. Therefore, two silver epoxy lines were applied on the glass substrate, which was able to fix the PVA nanofibers during the electrospinning process, as shown in Fig. 2(b). Fig. 2(c) shows, the multilayer PVA nanofibers were fabricated on the glass substrate, and a strong connection occurred between PVA nanofibers and silver epoxy after it cures. 


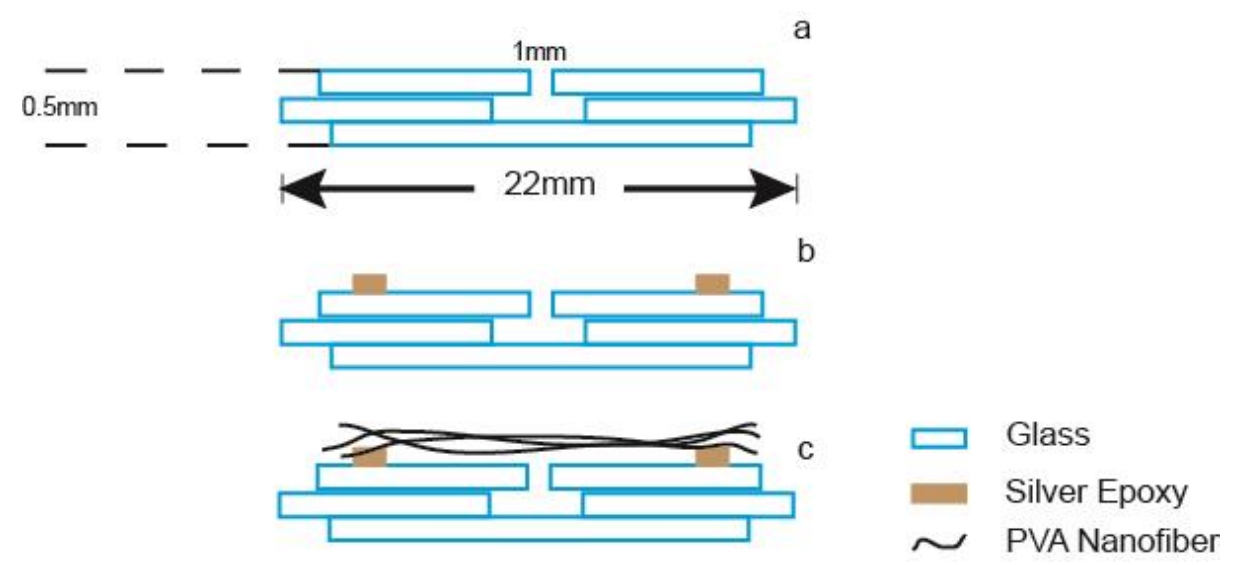

Fig. 2. Fabrication process of the multilayer PVA nanofibers on a unique glass substrate

\subsection{Palladium nanofibers fabrication}

Fig. 3 illustrates the fabrication process of the palladium nanofibers based on multilayer PVA nanofibers. The multilayer PVA nanofibers on a glass substrate was produced prior the palladium deposition process. According to the experiment design, the shadow mask was required to control the deposition area of palladium. Therefore, to avoid the cross-contamination between shadow mask and PVA nanofibers, the shadow mask was cleaned in ethanol and using ultrasonic. The shadow mask was a thin plastic sheet, the laser cutter (TRoTec Speedy $300^{\mathrm{TM}}$ ) was applied to cut the plastic sheet into $30 \mathrm{~mm} \mathrm{x}$ $30 \mathrm{~mm}$ square, with $15 \mathrm{~mm} \times 15 \mathrm{~mm}$ squire of window in the middle of the plastic sheet. During the palladium deposition process, the glass substrate was fully covered by shadow mask, as shown in Fig. 3(a).

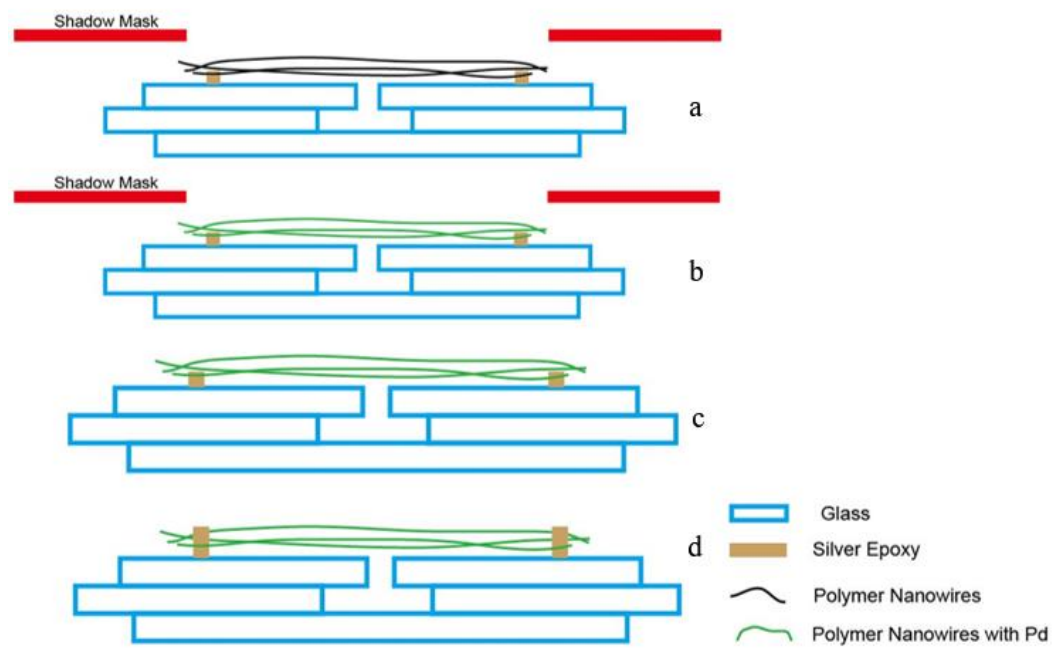

Fig. 3. Fabrication process of the palladium nanofibers based on multilayer PVA nanofiber 
The palladium deposition process was an important section for the fabrication of palladium nanofibers-based hydrogen sensor, and was implemented using E-beam evaporation system (Kurt J. Lesker) to achieve superior film density, and low impurity. The higher density should enhance the mechanical properties of palladium and increase the abrasion resistance. Palladium target with purity of $99.95 \%$ was installed in the crucible as an evaporation material. The coating growth was controlled by a quartz crystal microbalance which can monitor both thickness and evaporation rate. During the deposition process, the inside of the system must be in a vacuum state. The working temperature during this process at $25^{\circ} \mathrm{C}$ and $1 \times 10^{-8}$ Torr for the operation pressure. To further enhance density and smoothness palladium nanofibers, the deposition rate should be regulated as slow as possible. In this experiment, the evaporation rate was set at $1 \AA / \mathrm{s}$, and the sample was deposited $5 \mathrm{mins}$ to fabricate $30 \mathrm{~nm}$ palladium nanofibers as illustrated in Fig. 3(b). Then the palladium nanofibers were remained after removal of the shadow mask as shown in Fig. 3(c). Subsequently, the both electrodes of the sensor were made from silver epoxy, which was able to provide better electrical connection between electrodes and hydrogen sensing element, as shown in Fig. 3 (d).

\subsection{Characterization of the PVA nanofibers}

Scanning electron microscopy (SEM) was performed on the generated PVA nanofibers. The clean and smooth surface of the PVA nanofibers were demonstrated in Fig. 4. The random and continuous PVA nanofibers was presented in Fig. 4(a). Due to the different brightness in Fig. 4(b), the multilayer PVA nanofibers with diameters up to $500 \mathrm{~nm}$ were proved.

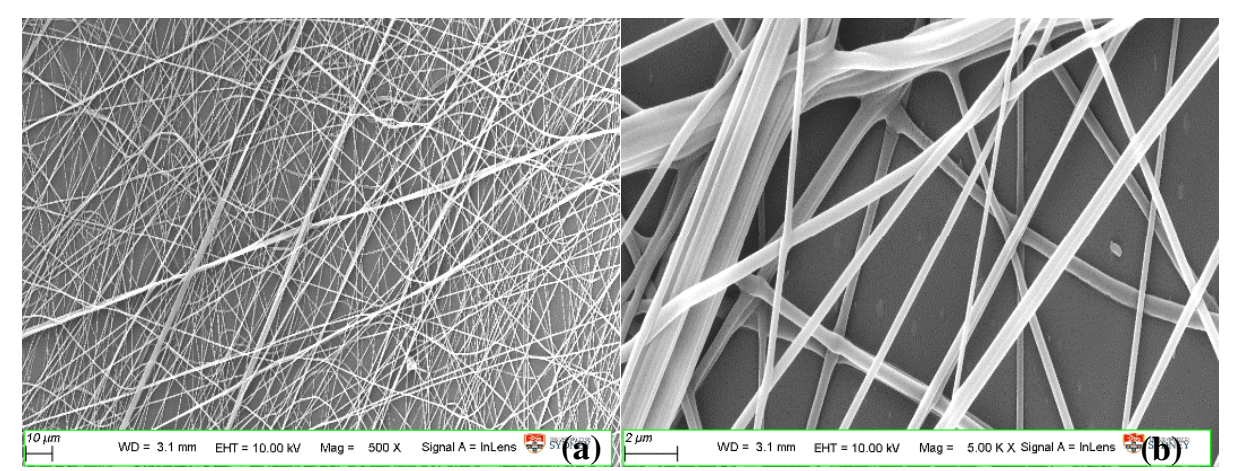

Fig. 4. SEM image of the surface morphology of a multilayer PVA nanofibers

\subsection{Hydrogen sensor testing system}

The hydrogen gas sensor was tested in a particular gas chamber (linkam Instruments), which was designed for cell and gas testing. The inside probes are positioned inside of this chamber making it ideal for resistance measurements when the sensor is exposed to hydrogen. The curing time for both electrodes were approximately $24 \mathrm{~h}$ at room temperature or 30 mins at $100{ }^{\circ} \mathrm{C}$. The electrodes became hard with high electrical 
conductivity after curing. Fig. 5 depicts a schematic diagram of the hydrogen gas testing system. Technically, the mass flow controller can produce various concentrations of hydrogen gas. During the gas detection test, $1 \%$ of hydrogen with nitrogen was produced via mass flow controller and discharged continuously into the gas chamber with a set flow rate of $300 \mathrm{~mL} / \mathrm{min}$ in standard condition. The valve of the mass flow controller was turned off after the measurement, and the outlet was opened to remove the remaining hydrogen gas. A PC connected with a digital multi-meter was applied for all measurement data collection and analysis. The change of resistance with hydrogen was recorded by digital multi-meter with time intervals of $1 \mathrm{~s}$. The gas response $\left(\mathrm{G}_{\mathrm{r}}\right)$ is defined as the percentage of resistance change when the palladium nanofiber hydrogen sensor is exposed to hydrogens.

$$
G_{r}=\frac{R_{b}-R_{a}}{R_{a}} \times 100 \%=\frac{\Delta R}{R_{a}} \times 100 \%
$$

Where $R_{a}$ and $R_{b}$ are the resistance of hydrogen sensor device before and after its exposure to the hydrogen concentration, respectively.

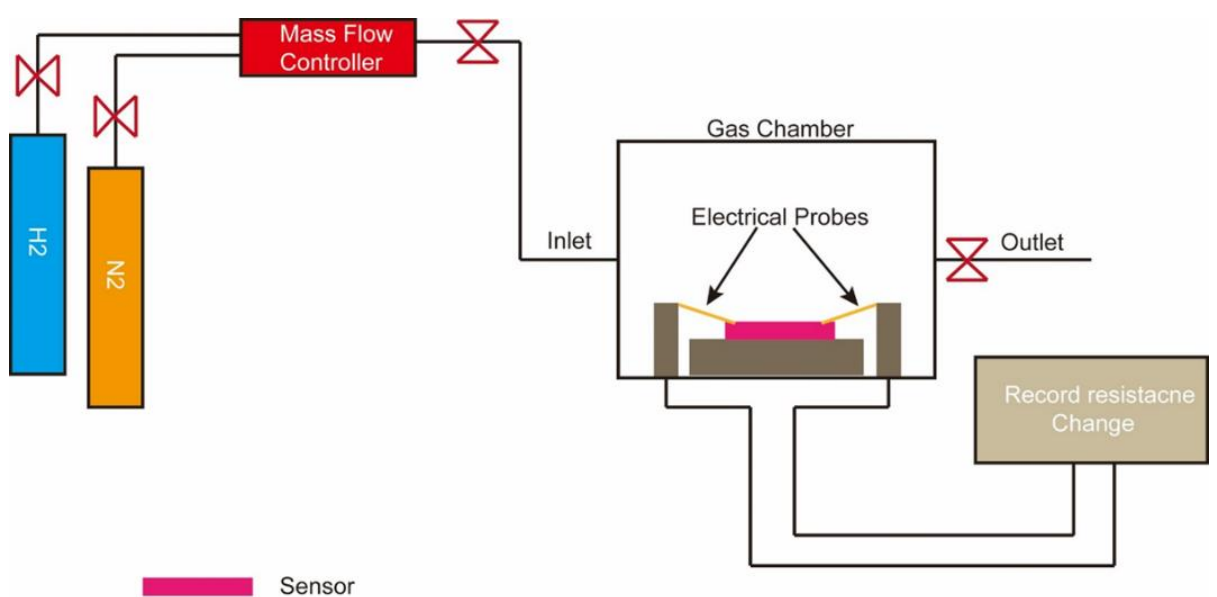

Fig. 5. Hydrogen gas testing system

\section{Results and Discussion}

\subsection{Morphology of the suspended palladium nanofibers}

As Fig. 6 presented, the palladium was coated on the surface of the multilayer PVA nanofibers. The PVA nanofibers were able to support $30 \mathrm{~nm}$ thick of palladium, but fractures were found in few parts of the nanofibers. The multilayer PVA nanofibers was exposed under palladium atoms with high energy during the deposition process. Therefore, they were damaged by high energy palladium atoms. Due to the fracture 
issues, the palladium nanofibers were not a continuous nanostructure. In the previous research, the surface fracture was able to influence the electrical resistivity property, sensitivity of hydrogen gas, and response speed. Specifically, the resistance of the palladium nanofibers was increased because of the fracture phenomena. However, the expansion of palladium lattice occurred when it was exposed to the hydrogen, and the resistance of the hydrogen sensor was decreased.

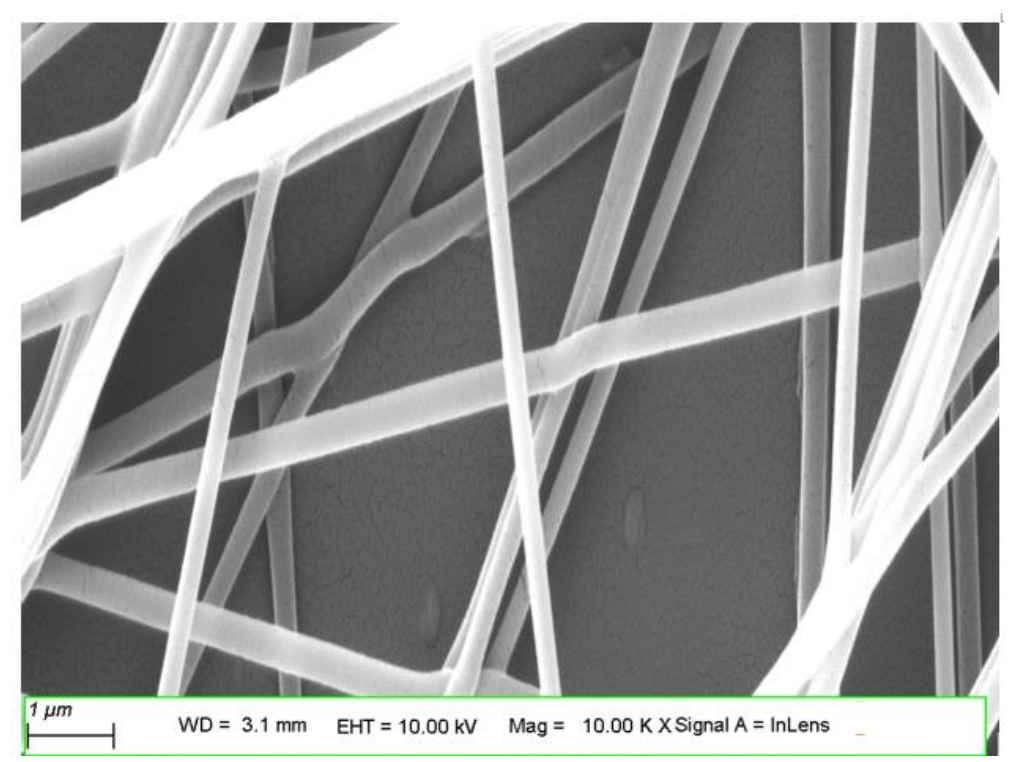

Fig. 6. SEM image of the surface morphology of the palladium nanofibers after gas detection process

\subsection{Hydrogen detection test}

The result in Fig. 7 indicated that the gas response for $1 \%$ hydrogen gas. According to the surface morphology of the palladium nanofibers, the superior material property of palladium nanostructure was demonstrated. The initial resistance of the hydrogen sensor was higher than normal palladium-based hydrogen sensors. Obviously, the trend of the gas response was different compare with others hydrogen sensors. As shown in Fig. 7, the resistance decreased when hydrogen was discharged into gas chamber, and it was increased when it was stopped. As discussed in subsection (3.1), the hydrogen sensor had undergone $\alpha$ to $\beta$ phase transition after absorbing hydrogen, and the facecenterd cubic (FCC) palladium lattice was expanded. The compressive stress on each Pd nanofibers are occurred, and noticeable compression were observed at grain boundaries. According to this characteristic, the continuous Pd nanofibers were generated and observed to have a decreased resistance. Conversely, removal of the Pd nanofibers to an air ambient induces a $\beta$ to $\alpha$ phase transition causing each palladium grains to contracted during the phase transition. This lead to an increased tensile stress on each Pd nanofibers, and resulted as nanoscopic gaps. The gaps generated when the 
sensor exposed to air ambient acted as open circuits which increased the resistance of the Pd nanofibers [16]. Collectively, the hydrogen sensing element was effectively an open electric circuit when it was exposed to air. The expansion of Pd lattice structure was able to lead the circuit became a closed circuit.

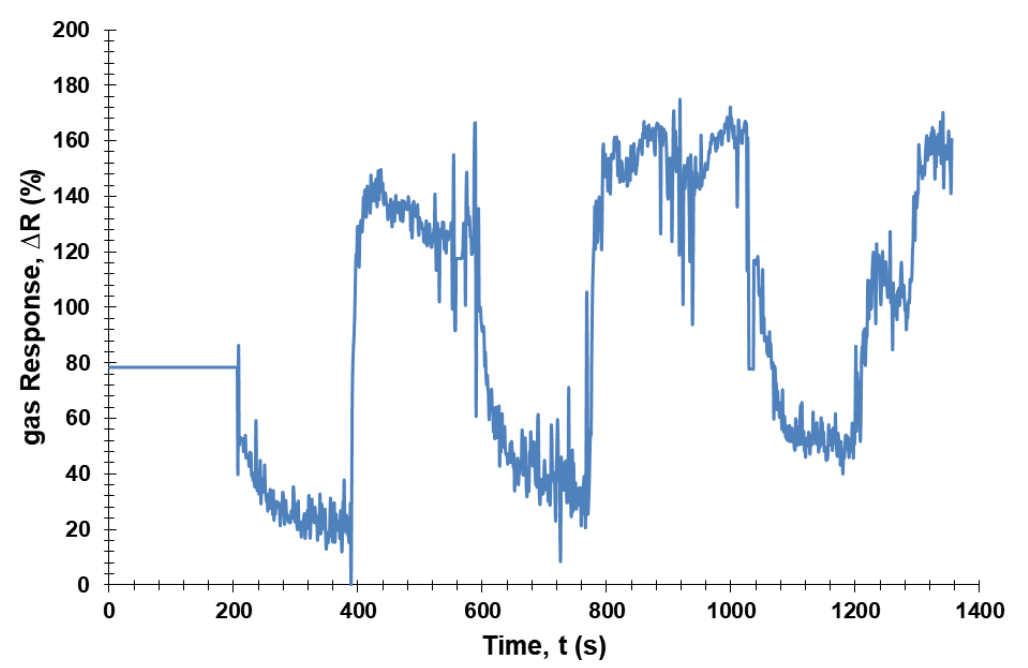

Fig. 7. $30 \mathrm{~nm}$ palladium nanofibers with $500 \mathrm{~nm}$ diameter PVA nanofibers, under $1 \%$ hydrogen testing

The response time is defined as the required time from initial point of $90 \%$ of the stable point, and the recovery time is defined as the required time from stable value to $10 \%$ of the stable value when hydrogen gas stops to introduce into gas chamber. As show in Fig 7, the response and recovery times at $1 \%$ hydrogen concentration were $92 \mathrm{~s}$ and 26 s, respectively. Meanwhile, the gas response was $78.57 \%$, approximately.

\section{Conclusions}

In this study, a hydrogen gas sensor based on palladium nanofiber networks was fabricated. The nanostructure was produced by electrospinning system and the multilayer PVA nanofibers was fabricated as a substrate, to simplify the fabrication process of palladium nanofiber networks. The E-beam evaporation technique was applied to deposit the $30 \mathrm{~nm}$ thick of Palladium on the surface of the substrate. Furthermore, the shadow mask was utilized to limit the sensing area of the hydrogen sensor. The proposed hydrogen sensor was able to detection hydrogen with $92 \mathrm{~s}$ response time, and $26 \mathrm{~s}$ recovery time, respectively. The gas response for the novel hydrogen sensor was approximate $78.57 \%$. In addition, the hydrogen sensor has become a hydrogen-actuated switches, due to the phase transition of palladium nanofibers. The fabrication of this novel hydrogen sensor is simple, and economical for mass-produced. 


\section{References}

1. Hübert,T., Boon-Brett,L., Black,G., Banach,U.:Hydrogen sensors - a review. Sens.Actuators B: Chem. 157, 329-352 (2011).

2. Lim, S, H., Radha, B., Chan, J,Y., Saifullah, M, S.M., Kulkarni, G, U.,Ho, G, W.: Flexible Palladium-based $\mathrm{H}_{2}$ Sensor with Fast Response and Low Leakage Detection by Nanoimprint Lithography. ACS Appl. Mater. Interfaces 5, 7274-7281(2013).

3. Zeng, X,Q., Wang, Y,L., Deng, H., Latimer, M,L., Xiao, Z,L., Pearson, J., Xu, T., Wang, H,H., Welp, U., Crabtree, G,W., Kwok, W,K.: Networks of Ultrasmall Pd/Cr Nanowires as High Performance Hydrogen Sensors. ACS NANO 5(9), 7433-7452(2011).

4. Lee, J., Zhu, Y., Seshia, A., Room temperature electrometry with SUB-10 electron charge resolution, Journal of Micromechanics and Microengineering, vol.18, no.2, 025033 (2008).

5. Zhu, Y., Lee, J., Seshia, A., A resonant micromachined electrostatic charge sensor, vol.8, no. 9, 1499-1505 (2008)

6. Jalil, J., Zhu, Y., Ekanayake, C., Ruan, Y., Sensing of single electrons using micro and nano technologies: a review, Nanotechnology, vol.28, no.14, 142002 (2017).

7. Lewis, F,A.: The Palladium-Hydrogen System. Platinum Metals Rev. 26(1),20-27(1982).

8. Lee,E., Lee,J.M., Koo,J.H., Lee,W., Lee,T.: Hysteresis behaviour of electrical resistance on $\mathrm{Pd}$ thin films during the process of absorption and desorption of hydrogen gas. International Journal of Hydrogen Energy 35, 6984-6991(2011).

9. Liu,Y.,Chen,Y.P.,Song,H.,Zhang,G.: Modelling analysis and experimental study on the optical fiber hydrogen sensor based on Pd-Y alloy thin film. Rev. Sci.Instrum. 83, 075001075005(2012).

10. Wang,B., Zhu,Y.,Chen,Y.P.,Song,H.,Huang,P.C.,Dao,D.: Hydrogen sensor based on palladium-yttrium alloy nanosheet. Meterials Chemistry and Physics 194, 231-235(2017).

11. Nguyen, T., Dinh, T., Phan, H., Tran, C., Foisal, A., Zhu, Y., Dao, D., Electrically Stable Carbon Nanotube Yarn Under Tensile Strain, IEEE Electron Device Letters, vol.38, no.9 1331-1334 (2017).

12. Phan, H., Dao, D., Wang, L., Dinh, T., Nguyen, N., Qamar, A., Tanner, P., Dimitrijev, S., Zhu Y., The effect of strain on the electrical conductance of p-type nanocrystalline silicon carbide thin films, Journal of Materials Chemistry C, vol.3, no.6, 1172-1176(2015).

13. Offermans,P., Tong,H.D., Van Rijn,C.J.M., Merken,P., Brongersma,S.H., CregoCalama,M.: Ultralow-power hydrogen sensing with single palladium nanowires. Applied Physics Letters 94, 223110(2009).

14. Lim, Y.,Lee,Y.,Heo,J.,Shin,H.: Highly sensitive hydrogen gas sensor based on a suspended palladium/carbon nanowire fabricated via batch microfabrication processes. Sens.Actuators B: Chem. 210, 218-224(2015).

15. Zeng, X.Q., Latimer, M.L., Xiao, Z.L., Panuganti, S., Welp, S., Kwok, W.K., Xu, T.: Hydrogen Gas Sensing with Networks of Ultrasmall Palladium Nanowires Formed on Filtration Membranes. NanoLetters 11, 262-268(2011).

16. Walter, E.C., Favier, F., Penner, R.M.: Palladium Mesowire Arrays for Fast Hydrogen Sensors and Hydrogen-Actuated Switches. Anal. Chem. 74, 1546-1553(2002). 